\title{
EL ARTE Y LA UNIVERSIDAD
}

\author{
P O R \\ X A VIER MOYSSEN
}

VERAz medida para valorar el desarrollo intelectual que ha alcanzado un pueblo suele ser la importancia que dentro de la colectividad nacional tenga su universidad, o por mejor decirlo, el grupo de instituciones universitarias con que cuente; y nada acreditará mejor la presencia de ese pueblo, dentro del concierto de las naciones cultas, que la madurez adquirida en los claustros universitarios, pues por esencia la universidad es, para los pueblos, la conformadora de su carácter, una poderosa fuerza de disciplina que coadyuva en la conquista y el progreso, y es, ante todo, la eterna fuente espiritual mediante la cual habrá de proyectarse siempre lo nacional hacia lo universal, que es a lo menos a que puede aspirar toda cultura.

Uno de los aspectos más nobles y valiosos que la Universidad $\mathrm{Na}$. cional Autónoma de México ha favorecido, con la más absoluta conciencia de su destino, es el relacionado con el arte mexicano, ya sea ante las obras de un pasado esplendoroso que en nada le es ajeno por ser depositaria del mismo, o bien ante las obras artísticas de un presente del cual ha sabido ser oportunamente, portavoz y gufa en el sentido más humano y universal. Hablar de esta labor constructiva de la Universidad en relación con el arte, y durante los cincuenta años que han transcurrido desde su reinstalación, por el Maestro don Justo Sierra, es pues, el tema de este trabajo.

La reinstalación de la Universidad Nacional se llevó a efecto, con toda la solemnidad que tal acontecimiento requería, el día 22 de sep- 
tiembre de 1910. Y la inauguración fue, a no dudarlo, uno de los actos más solemnes y trascendentales de las fiestas conmemorativas del primer centenario de la Independencia Patria. Ante los representantes de diversas universidades extranjeras que concurrieron a ver el renacimiento de esta casa de estudios, que volvía a la vida bajo el patrocinio de las de Salamanca, Paris y California, el Maestro Justo Sierra, en su carácter de Secretario de Instrucción Pública y Bellas Artes, pronunció el discurso inaugural, el cual, por las ideas de su contenido, ha pasado a ser una de las oraciones académicas más notables que se hallan pronunciado en México, a decir de la autorizada opinión del doctor Edmundo O'Gorman.

Reinstalada la Universidad, los estudios sobre manifestaciones estéticas habrian de impartirse en la Escuela de Bellas Artes, en la cual se acordó, por cierto, que solamente se enseñaría la arquitectura, excluyendo, por tanto, las disciplinas sobre pintura, escultura y grabado, que seguirian atadas a la vieja Academia de San Carlos. Sin embargo, los estudios sobre la historia del arte no quedarian excluidos de la enseñanza académica en la nueva Universidad, pues en una de sus dependencias más importantes, en la Escuela Nacional Preparatoria, don Manuel G. Revilla y el maestro don Carlos Lazo enseñaban con erudita paciencia y métodos cuasi positivistas la Historia Universal del Arte, cátedra que más tarde habría de pasar a la Escuela de Altos Estudios y cle ésta a lo que es hoy en dia, la Facultad de Filosofia y Letras, en la que, desde 1935, los estudios sobre arte mexicano ocuparon la importancia debida, al fundar el inolvidable maestro don Manuel Toussaint, su cátedra de Arte Colonial en México.

La corta existencia de la Universidad se vió agitada profundamente apenas a los dos meses de su reinstalación. No olvidemos la fecha: 1910. Y la Universidad no podfa situarse al margen de los hechos vitales que. se iban a suceder, no podía quedar excluída, bajo ningún concepto, del vórtice renovador, de la gran catarsis que para la vida mexicana, fue el movimiento de la Revolución. Y así, en medio del fragor de las luchas: fratricidas, en múltiples y penosas vicisitudes de toda indole, siguió adelante en su destino, identificado ahora con los postulados de la revolución del pueblo, para el que mantenía viva, a pesar de todo, la universalidad de una cultura, de la que era depositaria y celoso guardián, al fin y al cabo. Y gracias a esa cultura la Universidad no tardó, en cuanto. las circunstancias fueron propicias, en dar justificación y prestigio al nuevo orden que trajo consigo la Revolución. 
Una de las aportaciones más valiosas que en el terreno de los valores culturales, a buena hora, tuvo la Revolución Mexicana, fue la pintura mural, poes ella representó, con gran categoría estética, las reivin. dicaciones y el mensaje de un pueblo que construía, para encontrarse a sí mismo. Pero no solamente eso, gracias a esta pintura mural la presencia artística de México ocupó un sitio singular dentro de las manifestaciones estéticas de la primera mitad de este siglo.

$Y$ tocó, nada menos que a la Universidad Nacional, ser la propiciadora de este arte de fuerte contenido humano y universal, como es la pintura de que hoy nos orgullecemos al presentarla. Siendo la Universidad el centro intelectual de mayor prestigio y tradición en el país, el. hecho de haber favorecido este movimiento artístico, tal vez nada tuvo de fortuito, pues conviene considerar los antecedentes que en materia de gran arte tuvo esta máxima casa de estudios en el siglo xIx; la referencia es el mural que en la Escuela Nacional Preparatoria pintó Juan Cordero en 1874, bajo el signo de Orden y Progreso, que desde esas aulas acuñara don Gabino Barreda, a quien su positivismo no impedía señalar y aún criticar en las palabras de su discurso, el por qué no se había "sancionado antes de una manera franca y solemne, la indispensable fraternidad (entre) la ciencia y la estética". I A pesar de que el mural de Cordero, aomo se dijo en su momento, "deleita a quien lo ve sólo de paso y pone pasmo y admiración en quien lo estudia y contempla detenidamente" 2 , no obstante lo dicho, más tarde habría de ser destruído para sustituirle con el grosero y prosaico emplomado industrial que hasta la fecha subsiste; vitral "anodino, elegido por medio de catálogo y encargado naturalmente a Europa" 3; el mural de Juan Cordero tiene pues, un valor significativo en el arte de un pueblo que suele manifestarse monumentalmente.

En 1910 el arquitecto Samuel Chávez, concluyó las obras del Anfiteatro, al que por entonces solamente se le llamó "de la Preparatoria";

1 Poesia y Discursos leidos en la festividad en que la Escuela Nacional Preparatoria, laureando al eminente artista $D$. Juan Cordero, le dio un testimonio público de gratitud y admiración, por el cuadro mural con que ha embellecido su edificio. México, Imprenta del Comercio, de N. Chávez, 1874, pág. 26.

2 Discurso pronunciado por el C. Profesor Rafael Angel de la Peña, a nombre de los catedráticos de la Escuela. Op. cit. pág. 3.

3 José Rojas Garcidueñas El Antiguo Colegio de San Ildefonso; I. de I. E. México, 1951, pág. 67. 
su decoración iba a quedar a cargo del gran pintor Saturnino Herrán, mas los acontecimientos inmediatos impidieron toda obra, de la que sin duda habria que esperar mucho, dada la personalidad del artista y cierta conciencia de renovación plástica que él posefa, misma que se manifiesta en sus estudios para el gran friso Nuestros dioses, que no logró pintar en el Teatro Nacional.

En 1921 José Vasconcelos, desde la Secretaria de Educación Pública, abrió las puertas de la Universidad a los artistas, ofreciéndoles los muros de la misma para que los pintaran, no importa que esto lo haya desconocido más tarde, el apasionado y discutido maestro, así como desconoció ideológicamente el contenido exacto del lema que diera a la Universidad, la cual, conviene tener presente, dependía por entonces de Educación Pública. El mural que Diego Rivera pintó con la técnica de la encáustica en 1922, en el Anfiteatro, fue la primera obra de trascendencia universal de la pintura mexicana contemporánea.

A la obra de Rivera, siguió la monumental labor artística de José Clemente Orozco, plasmada en los grandes muros del patio principal y escalera, de la Escuela Nacional Preparatoria, en los que quedó manifiesta la calidad estética del genial pintor, pues a decir, con la autoridad del doctor Justino Fernández, "pinturas al fresco de calidad tal, no se habian producido desde el Renacimiento". Mas lo importante es, continúa diciéndonos, "la potencia expresiva del artista para elevar a cate. gorias universales las circunstancias de la Revolución Mexicana y el profundo cambio vital de nuestros días, asi como la interpretación, por primera vez en la histonia de la pintura, del origen de la América Hispana en términos de su ser, (que) revelaron bien pronto la poesia épica que anima toda la obra del artista y su profundo sentido auténticamente humano. LA TRINCHERA es, por si, una de las grandes obras de todos iós tiempos, $y$, en suma, estos frescos son las pinturas más valiosas que guarda la Universidad" "4.

El venerable edificio de la Escuela Nacional Preparatoria, recogió en los grandes paños de sus muros, las primeras obras artísticas, con carácter monumental, de un buen número de pintores. En 1922 David Alfaro Siqueiros pintó, en la escalera del Patio Chico, unos interesantes murales con la acusada fuerza expresiva y emoción plástica que le dis-

4 Véase: "La Pintura Contemporánea y la Universidad", en Ensayos sobre la Universidad de México, pág. 88. Ediciones del IV Centenatio de la Universidad de México. 1951. 
tingue. A Ramón Alva de la Canal y a Fermín Revueltas, ayudado por Máximo Pacheco, tocóles pintar los muros del cubo de la entrada principal que da a la vieja calle de San Ildefonso. El año de 1922 fue de una prodigalidad inaudita, pues aparte de las obras que pintaban los artistas anotados, en esa fecha el francés Jean Charlot, pintó su célebre mural sobre La toma de Tenoxtitlán por los españoles, y frente a éste, quedó el de Fernando Leal inspirado en un tema popular La fiesta del Señor de Chalma, y el mismo Leal, años más tarde -1933- se encargó de decorar el vestibulo del Anfiteatro Bolivar, con temas sobre la vida del gran héroe americano.

Como los artistas vivían la circunstancia histórica de la Revolución, con la que estaban identificados tanto en el pensamiento como en la acción, ya se comprende que en ella encontraron temas suficientes para sus murales; pero, así mismo, la realidad vital del pueblo que se descubría se presentó como fuente inagotable de inspiración.

Otros edificios ocupados también por dependencias universitarias, fueron enriquecidos con las pinturas murales de estos grandes artistas. En la Hemeroteca Nacional trabajaron, desde 1921, Roberto Montenegro, Xavier Guerrero y Jorge Enciso, y en lo que fue la Preparatoria Chica, hoy Escuela Secundaria número seis, el Doctor Atl pintó algunos murales en el patio principal, los que más tarde fueron destruídos por orden de Narciso Bassols, por considerarlos "completamente paganos", es decir, fuera de la temática habitual de contenido bolchevique que estaba en boga, según afirma el propio pintor."

Como se ha visto, la monumental pintura contemporánea de México, se inició y realizó en buena parte, gracias a la Universidad Nacional; pese a los múltiples problemas e incomprensiones que a cada momento surgían, el propio Orozco atestigua sobre las circunstancias en que fueron pintados muchos de esos murales ${ }^{\theta}$; mas conviene destacar el amplio espiritu liberal que reinó por parte de las autoridades univer. sitarias para que los artistas pintaran en absoluta libertad.

Respecto a la enseñanza del arte, desde los talleres y cátedras la labor de la Universidad ha sido de lo más positivo. La tradicional Academia de San Carlos, o Nacional de Bellas Artes, dejó de funcionar como tal desde 1929, pues por dificultades internas en los programas de enseñan.

5 Clementina Díaz y de Ovando, El Colegio Mdximo de San Pedro y San Pablo. I. de I. E. México, 1951, lámina $\$ 6$.

6 En su Autobiografia. Ediciones Occidente. México. 1945, págs. 111 a 113. 
7a, fue dividida para crear la Facultad de Arquitectura y la Escuela de Artes Plásticas, de la que Diego Rivera fue el primer director por una corta temporada. Con la nueva organización la Universidad perdió la custodia de las Galerías de Pintura y Escultura, mexicana y europea, que ahí se conservan desde el siglo xIx, y dió lugar, también, a que la Secretaría de Educación Pública abriera en 1943, la Escuela de La Esmeralda.

La Escuela de Artes Plásticas ha cumplido con su finalidad de manera generosa y eficaz, no obstante que en algunas ocasiones ha tenido que hacerlo en críticas circunstancias; por sus talleres de dibujo, pintura, escultura y grabado, han pasado los mejores maestros de la plástica mexicana contemporánea.

Un papel de primer orden es el que corresponde a la Facultad de Arquitectura, pues al afrancesamiento, estilo Ecole des Beaux Arts, de la arquitectura anterior, se opusieron desde 1923, la sabia disciplina y e] talento renovador de un grupo de arquitectos mexicanos, a cuya cabeza es justo colocar al maestro José Villagrán García; mas conviene no olvidar, también, a los arquitectos pioneros del nuevo movimiento: Juan O'Gorman, Juan Legarreta, Mauricio Campos, Enrique del Moral, Enrique Yáñez y Enrique de la Mora; y una gran trascendencia en esta tarea de renovación, fue la que tuvieron los célebres Talleres de Arquitectura que tanto ayudaron en la formación de las nuevas generaciones. La importante realidad arquitectónica del México que emergió de la Revolución de 1910, sólo ha sido posible gracias a los egresados de esa Facultad, y la muestra más rotunda y elocuente de ello es, sin duda, la Ciudad Universitaria, grandiosa y bella realidad en la que se conjugaron admirablemente las fuerzas seculares de un espiritu artístico, con las más novedosas formas constructivas y de expresión plástica. Es conveniente señalar aquí otras grandes obras arquitectónicas de alcance nacional: hospitales, escuelas y edificios multifamiliares, que no son otra cosa que las realizaciones más efectivas y plausibles de los gobiernos emanados de la Revolución. Y aún hay que agregar otros aspectos de esta arquitectura que da dignidad al país, me refiero a las grandes construcciones de empresas particulares, así como a los edificios de las nuevas zonas residenciales; la planificación ocupa también un sitio de singular importancia y lo mismo hay que decir respecto a la arquitectura destinada al culto religioso que cuenta con novedosas y atractivas realizaciones.

Pero la enseñanza del arte no se ha limitado únicamente a los dos planteles que se han citado, hay otro aspecto digno de considerarse: las 
cátedras de Historia del Arte que en la Facultad de Filosofía y Letras imparte un grupo de maestros distinguidos; yo deseo destacar, en esta ocasión, tan sólo las dedicadas al estudio del arte mexicano. Ya se ha dicho que la primera fue dedicada al Arte Colonial desde 1934, por don Manuel Toussaint y hoy en día a cargo del doctor Francisco de la Maza. La cátedra de Arte Precolombino, fue fundada en 1945 por Salvador Toscano, si bien anteriormente la dictaba en la Escuela de Artes Plásticas; el curso desde 1950, está a cargo del profesor José Servín Palencia; y finalmente, la cátedra de Arte Moderno y Contemporáneo, fue establecida en 1945 por el doctor Justino Fernández.

La trascendencia y valor que han tenido estas tres cátedras para la difusión y conocimiento del arte mexicano, ha sido una de las labores más positivas que se han hecho desde esta Facultad. $Y$ hay que anotar, también, la importancia que han tenido estos mismos cursos dictados para los estudiantes extranjeros al través de la Escuela de Verano, fundada en 1921 por el maestro Pedro Henriquez Ureña.

La investigación como tarea educativa era una de las preocupaciones de don Justo Sierra, asi lo manifestó en su célebre Discurso inaugural de la Universidad, decia ahi "... todo lo que es necesario proteger... exige la creación temporal de medios facticios favorables a esa evolución que tenemos por indispensable a la cultura nacional, me refiero a las enseñanzas estéticas, (que) quedan en nuestro plan pedagógico... nues. tra ambición sería que... se enseñase a investigar, investigando y pensando..." T Este interés que demostró don Justo Sierra, en realidad venía a responder a una urgencia, a una necesidad de índole intelectual que "andaba en el espiritu de la época", tal por lo menos habia manifestado ya, el Ministro de Justicia del régimen porfirista, licenciado don Justino Fernández.

El problema de la investigación estética dentro de la Universidad vino a quedar resuelto ampliamente veinticinco años después, en 1935, cuando bajo la dirección de don Manuel Toussaint se estableció un La. boratorio de Arte, a semejanza del ya existente en la Universidad de Sevilla. Las investigaciones sistemáticas sobre el arte mexicano, nacieron all, pues con plena conciencia don Manuel Toussaint aseveraba en un oficio enviado al Rector, doctor Fernando Ocaranza, lo siguiente: " $L a$ Historia de nuestras artes plásticas está por hacerse. Ha habido estima.

7 Justo Sierra Prosas. Biblioteca del Estudiante Universitario. Tomo 10. México, 1939., ver págs. 191 y 193. 
bles esfuerzos aislados, pero falta un centro coordinador y autorizado. Este puede y debe ser nuestra Universidad, centro moximo de cultura en el pais". En 1936 con la reorganización que hiciera en la Universidad el Rector Luis Chico Goerne, el Laboratorio de Arte se transformó en Instituto de Investigaciones Estéticas el cual, salvo una breve interrupción de labores en 1938, ha trabajado incansablemente desde entonces, difundiendo los valores del arte mexicano gracias a las investigaciones realizadas por sus miembros.

Estas investigaciones son difundidas, aparte algunas conferencias, de manera preferente en las acreditadas publicaciones del propio Instituto, el cual cuenta en su haber con la publicación de las obras fundamentales para el estudio del arte mexicano, de las que tan sólo habré de citar en esta ocasión, una parte minima. En las publicaciones seriadas, el primer sitio lo ocupan los "ANALES" del Instituto; el número 19 apareció en 1937 y a la fecha el número 30 habla de una labor fecunda y constante. "Estudios y Fuentes del Arte en México" es una interesante colección en la que se recogen en trabajos monográficos, diversos aspectos del arte, tales como el teatro, el arte popular, el colonial y el contemporáneo, en tanto que en la Serie "Estudios de Arte y Estética" quedan incluidos trabajos de gran significación estética en la Historia de las Ideas, asi se ha publicado El Arte y la Estética del Budismo de Jean M. Riviere; De la Belleza en el Arte Clásico de Winckelmann, en la esmerada traducción del doctor Juan A. Ortega y Medina, ilustre catedrático de la Facultad de Filosofía y Letras; del doctor Justino Fernández, actual director del Instituto, se han publicado dos estudios fundamentales sobre arte mexicano, el primero es su clásico tratado sobre el arte indígena antiguo: Coatlicue, en tanto que en el segundo, que lleva el título significativo de El Retablo de los Reyes, se ocupa de las manifestaciones artísticas del México virreinal. Otras obras merecen mencionarse, tanto por el valor de su contenido como por la esmerada calidad tipográfica que con gala de buen gusto hay en ellas; la reedición del libro de Claudio Linati Trajes civiles, militares y religiosos de México (1828), figura a la cabeza; otros libros son, el estudio sobre El Gran Signo Formal del Barroco, de Víctor Manuel Villegas; La Sillería de San Agustín, con una introducción de don Rafael García Granados; de Luis Cardoza y Aragón su monografía Orozco. Para la celebración del Cuarto Centenario de la Universidad, el Instituto colaboró con la publicación de una interesante serie de monografias, escritas por sus investigadores, sobre 
los monumentos arquitectónicos que ocupaban algunas escuelas universitarias.

La investigación referente a los valores de la literatura nacional, no ha sido olvidada por el Instituto, pues aparte de los valiosos estudios que han aparecido en diversos números de los Anales, en 1958 se dió a luz la paciente y valiosa investigación de José Rojas Garcidueñas, sobre la noble figura de Bernardo de Balbuena. Un espléndido estudio sobre el mérito literario de Juan Díaz Covarrubias, fue elaborado por la invesrigadora Clementina Díaz y de Ovando, para publicarse junto con las Obras Completas del malogrado escritor romántico. La rica veta del folklore, y de idéntica manera el teatro del siglo xIX, han sido estudiados prolijamente por don Vicente T. Mendoza y por Luis Reyes de la Maza.

Pero hay una magna obra que por sí misma justifica la existencia del Instituto de Investigaciones Estéticas, ésta es, nada menos, que la primera gran historia del arte mexicano, obra escrita por las máximas autoridades en la materia: a Salvador Toscano débese el tomo sobre Arte Precolombino de México y América Central, publicado en 1944, El Arte Colonial en México, a don Manuel Toussaint, apareció en 1948, y el tercer tomo, publicado en 1952, en el que se estudia el Arte Moderno y Contemporáneo de México, corresponde al doctor Justino Fernández. Obra valiosa, si las hay, es esta importante historia del arte mexicano.

El prestigio que ha adquirido el Instituto por la honradez y calidad de sus trabajos, ha traspasado las fronteras nacionales, y otras instituciones a imitación suya, han nacido en Bolivia y Argentina, en este último pafs, incluso, lleva una denominación casi semejante: Instituto de Arte Americano e Investigaciones Estéticas, y publica, igualmente, sus Anales.

Y a propósito de libros de arte, nada mejor que indicar aquí las obras que han salido de las prensas universitarias, desde aquellas publicadas con el rubro de Imprenta Universitaria, hasta las de hoy en día de la Dirección General de Publicaciones. Entre las primeras ediciones hay que anotar el ensayo de Luis Cardoza y Aragón sobre pintura contemporánea, La Nube y el Reloj; más tres monograffas: Imagineria Co. lonial de Manuel Toussaint; Asúnsolo, Escultura Mexicana Contemporánea de Juan de la Encina, y Manuel Rodriguez Lozano, con texto de José Bergamín. Débese a la Dirección General de Publicaciones, entre otras obras, la Colección de Arte, dirigida por Pedro Rojas, misma que cuenta ya con siete títulos, a cual más de ellos de positivo interés; en un 
portafolio se recogieron los preciosos dibujos de animales del artista Héctor Xavier, con el título Punta de Plata, y recientemente ha salido de las prensas el libro de Miguel Covarrubias Arte Indigena de México $y$ América Central. Como se ve ningún aspecto del arte mexicano se ha descuidado.

De otras manifestaciones de las bellas artes, también se ha ocupado la Universidad; el teatro y la danza no le han sido ajenos ni mucho menos, pues cuenta con escuelas donde se estudian y practican esas disciplinas; y algo semejante puede decirse respecto a la música de los grandes maestros.

El pasado artístico de México con entera conciencia lo protege la Universidad Nacional, máxime cuando ese legado lo ha recibido en forma viva, en vetustos y venerables edificios desde los cuales se ha im. partido la universalidad del saber; entre las obras de protección a ese pasado artístico es digna de anotarse la reconstrucción de una de las obras más extraordinarias de la escultura virreinal: la famosa sillerfa del coro de San Agustín, instalada en "El Generalito" de la Escuela Nacional Preparatoria.

Las galerías de arte, dependientes de la Universidad, han cumplido satisfactoriamente con la misión para que fueran creadas. En 1937 y organizada por el entonces Departamento de Acción Social, se fundó la Galeria de Arte, bajo la dirección de Julio Castellanos; ahí se presentó con gran éxito una de las primeras exposiciones de arte mexicano contemporáneo. En el vestíbulo de la Biblioteca Nacional, acondicionado para galeria, también se presentaron interesantes exposiciones mediante las cuales el pueblo se puso en contacto con las obras de sus grandes artistas. Hoy en día esta función la desempeña, con creces, el Museo instalado en la Ciudad Universitaria, en donde se han mostrado exposiciones de gran interés, la de Arte Peruano merece destacarse entre otras.

Ha tocado, en los últimos años, a la Dirección General de Difusión Cultural, ejecutar una de las misiones más valiosas que se han puesto en práctica para el conocimiento del arte mexicano, cual es el mostrarlo nada menos que en sus sitios de origen; eso ha sido posible mediante una larga serie de excursiones culturales que dirigen maestros universitarios y en las que se visitan desde los centros arqueológicos más distantes y de difícil acceso, hasta los edificios de esta Capital que se engalanan con las pinturas murales de Orozco, Tamayo, Rivera y Siqueiros, sin olvidar, claro está, las monumentales obras arquitectónicas del arte novo. español, situadas en los cuatro puntos cardinales de la rosa de los vien- 
tos, de la suave patria a la que cantara López Velarde. Esta encomiable labor, primera en su género, no es la única empresa a la que se dedica la Dirección General de Difusión Cultural; en sus actividades cuentan los conciertos de música clásica, representaciones de danza y teatro, re. citales de poesía, así como el patrocinio de exhibiciones de Cine-Club; toda una labor de difusión como se ve.

Con la exposición de este trabajo se han mostrado, en una visión de conjunto, las diversas actividades que la Universidad Nacional ha desarrollado, respecto al arte de México, durante los primeros cincuenta años de su reinstalación. Guárdase la convicción de que ha cumplido satisfactoriamente con uno de los cometidos culturales que más le dignifican y enaltecen. 